\title{
Alternative approach to general coupled linear optics
}

\author{
Andrzej Wolski* \\ Lawrence Berkeley National Laboratory, Berkeley, California 94720, USA
}

(Received 29 November 2005; published 3 February 2006)

\begin{abstract}
The Twiss parameters provide a convenient description of beam optics in uncoupled linear beam lines. For coupled beam lines, a variety of approaches are possible for describing the linear optics; here, we propose an approach and notation that naturally generalizes the familiar Twiss parameters to the coupled case in 3 degrees of freedom. Our approach is based on an eigensystem analysis of the matrix of secondorder beam moments, or alternatively (in the case of a storage ring) on an eigensystem analysis of the linear single-turn map. The lattice functions that emerge from this approach have an interpretation that is conceptually very simple: in particular, the lattice functions directly relate the beam distribution in phasespace to the invariant emittances. To emphasize the physical significance of the coupled lattice functions, we develop the theory from first principles, using only the assumption of linear symplectic transport. We also give some examples of the application of this approach, demonstrating its advantages of conceptual and notational simplicity.
\end{abstract}

DOI: 10.1103/PhysRevSTAB.9.024001

PACS numbers: 29.20.Dh, 29.27.Bd

\section{INTRODUCTION}

Linear optics in uncoupled beam lines are conventionally described by means of the Twiss parameters. The Twiss parameters or lattice functions relate the beam distribution in phase space at any point in a beam line to conserved quantities - the emittances - that are properties of a bunch traveling along the beam line. For example, the horizontal mean square beam size $\left\langle x^{2}\right\rangle$ is related to the horizontal emittance by $\left\langle x^{2}\right\rangle=\beta_{x} \epsilon_{x}$, where $\beta_{x}$ is the horizontal Twiss beta function that varies along the beam line, and $\epsilon_{x}$ is the horizontal emittance (a conserved property of the beam). Coupling in a beam line can transfer motion from 1 degree of freedom into another; this can arise, for example, from the presence of skew quadrupoles, or from RF cavities at locations of nonzero dispersion.

Two approaches for describing the transverse optics in coupled beam lines are well known. In one approach, introduced by Edwards and Teng [1] and developed by others [2-4], coupled betatron motion is analyzed by defining a "decoupling transformation" that puts the $4 \times$ 4 transfer matrix into block-diagonal form. The lattice functions are obtained from the block-diagonal components of the transformed transfer matrix by a procedure similar to that used for uncoupled motion. Additional functions to describe the coupling are obtained from the decoupling transformation. A disadvantage of this technique is that the lattice functions are not directly related to the beam sizes. Also, the procedure does not easily generalize to more than 2 degrees of freedom.

In the second approach, a transformation is found from the eigenvectors of the transfer matrix, that puts the transfer matrix into "normal form," i.e., the transfer matrix is transformed into a pure rotation. The lattice functions are

*Electronic address: awolski@lbl.gov defined in terms of elements of the normalizing transformation. This is the basis of the approach used by Ripken $[5,6]$ and by Lebedev and Bogacz [7]. These authors minimize the number of lattice functions used to describe the optics, and as a result the interpretation of the various lattice functions is not as simple as it might be. Also, the theory is developed only for motion in 2 degrees of freedom. However, in an accelerator, the coupling between transverse and longitudinal motion (described by the dispersion) is often not negligible, and it is desirable to have a description of the optics that applies as naturally and easily to 3 degrees of freedom as to 2 .

In this paper, we develop a description of coupled linear optics that addresses issues inherent in previous approaches. In particular, we aim to develop a description of the optics that maintains the conceptual simplicity of the uncoupled case (by providing lattice functions that directly relate the beam emittances to the beam distribution in phase space), and is capable of describing motion in any number of degrees of freedom with equal ease. We start from the eigenvector analysis, but differ from previous work in that we do not aim to reduce the number of lattice functions to the absolute minimum. We feel that it is more useful to have functions that have clear physical significance, than to know that all the functions in the theory are truly independent of one another. Since there is a natural systematic notation in our approach that emphasizes the physical significance of the lattice functions, it is easy to keep track of the various functions and to manipulate expressions in which they appear. Our approach is general in that we make no assumptions about the beam line (beyond requiring linearity and symplecticity) or about the form of the beam distribution (our analysis applies to Gaussian and non-Gaussian beam distributions).

We begin in Sec. II by making some general definitions. In Sec. III, we consider linear beam optics in storage rings, 
using the concept of a matched distribution to define a set of coupled lattice functions that directly relate the matched distribution to a set of conserved quantities - the beam emittances. We then consider in Sec. IV the propagation of the lattice functions along a transport line (nonperiodic beam line), and also obtain the phase advance along such a beam line. Finally, in Sec. V we give some practical examples that illustrate how our approach and notation provide a convenient way for describing coupled linear optics in storage rings and transport lines.

\section{GENERAL DEFINITIONS}

The beam distribution is described by a symmetric matrix $\Sigma$ of second-order moments. The components of $\Sigma$ are given by

$$
\sigma_{i j}=\left\langle x_{i} x_{j}\right\rangle
$$

where the brackets $\langle\cdot\rangle$ denote an average over all particles in the beam. The coordinates $x_{i}$ are components of the phase-space vector of a particle

$$
\mathbf{x}=\left(\begin{array}{c}
x \\
p_{x} \\
y \\
p_{y} \\
z \\
\delta
\end{array}\right) .
$$

We shall implicitly assume that the beam has vanishing first-order moments, i.e.,

$$
\left\langle x_{i}\right\rangle=0 .
$$

If necessary, it is straightforward to perform a transformation to variables in which this condition is satisfied.

The variables $\left(x, p_{x}\right),\left(y, p_{y}\right)$ and $(z, \delta)$ form three sets of canonical conjugate pairs. In this case, the linear transfer matrix between any two points in a beam line will be symplectic. Note that $z$ is the distance that a particle is ahead of the reference particle; $\delta$ is the relative energy deviation

$$
\delta=\frac{E-E_{0}}{E_{0}},
$$

where $E_{0}$ is the reference energy.

For convenience we define some $2 \times 2$ matrices

$$
\begin{aligned}
& \mathbf{0}_{2}=\left(\begin{array}{ll}
0 & 0 \\
0 & 0
\end{array}\right), \\
& \mathbf{I}_{2}=\left(\begin{array}{ll}
1 & 0 \\
0 & 1
\end{array}\right), \\
& \overline{\mathbf{I}}_{2}=\left(\begin{array}{ll}
0 & 1 \\
1 & 0
\end{array}\right),
\end{aligned}
$$

$$
\begin{aligned}
& \mathbf{S}_{2}=\left(\begin{array}{cc}
0 & 1 \\
-1 & 0
\end{array}\right), \\
& \overline{\mathbf{S}}_{2}=\left(\begin{array}{cc}
-1 & 0 \\
0 & 1
\end{array}\right) .
\end{aligned}
$$

A matrix $\mathbf{M}$ is symplectic if it satisfies the condition

$$
\mathbf{M}^{\mathrm{T}} \mathbf{S M}=\mathbf{S},
$$

where $\mathbf{S}$ is the antisymmetric matrix

$$
\mathbf{S}=\left(\begin{array}{lll}
\mathbf{S}_{2} & & \\
& \mathbf{S}_{2} & \\
& & \mathbf{S}_{2}
\end{array}\right) .
$$

\section{STORAGE RINGS}

In this section, we consider the case of beam optics in a storage ring. This allows us to define the concept of a matched distribution, which will be used to specify the values of the lattice functions at each point in the ring. We begin by showing an equivalence between the eigenvectors of the single-turn linear map $\mathbf{M}$, and the eigenvectors of the matrix $\Sigma \mathbf{S}$ constructed from the beam distribution matrix. We then show that the beam emittances are the eigenvalues (within a factor $\pm i$ ) of $\Sigma \mathbf{S}$. This leads (via a definition of the action as a conserved quantity of the motion of a particle under linear symplectic transport) to a natural definition of the lattice functions as quantities that relate the matched beam distribution in phase space at any point in a storage ring to the beam emittances. We end this section by showing that in the special case of zero coupling, the lattice functions we derive in this way correspond to the familiar Twiss parameters.

\section{A. Eigenvectors of the single-turn matrix and the beam distribution matrix}

Since the linear single-turn map $\mathbf{M}$ is symplectic, it has six eigenvalues that can be arranged in reciprocal pairs

$$
\lambda_{-k} \lambda_{k}=1 \text {, }
$$

where $k=$ I, II, III. The eigenvectors $\mathbf{e}_{ \pm k}$ of $\mathbf{M}$ are defined by

$$
\mathbf{M e} \mathbf{e}_{ \pm k}=\lambda_{ \pm k} \mathbf{e}_{ \pm k},
$$

and are normalized so that

$$
\begin{aligned}
\mathbf{e}_{-k}^{\mathrm{T}} \mathbf{S e}_{k^{\prime}} & =i \delta_{k k^{\prime}}, \\
\mathbf{e}_{-k} & =\mathbf{e}_{k^{*}}^{*} .
\end{aligned}
$$

If the storage ring lattice is linearly stable, then the eigenvalues of the single-turn map will lie on the unit circle

$$
\left|\lambda_{k}\right|=1
$$


and the three tunes $\nu_{k}$ are given by

$$
\lambda_{ \pm k}=\exp \left( \pm 2 \pi i \nu_{k}\right) .
$$

We now construct the matrix $\mathbf{E}$ from the set of eigenvectors $\mathbf{e}_{ \pm k}$. Explicitly

$$
\mathbf{E}=\left(\begin{array}{llllll}
\mathbf{e}_{-I} & \mathbf{e}_{\mathrm{I}} & \mathbf{e}_{-\mathrm{II}} & \mathbf{e}_{\mathrm{II}} & \mathbf{e}_{-\mathrm{III}} & \mathbf{e}_{\mathrm{III}}
\end{array}\right) .
$$

With the normalization (14), $\mathbf{E}$ obeys the relation

$$
\mathbf{E}^{\mathrm{T}} \mathbf{S E}=i \mathbf{S}
$$

We note that

$$
\mathbf{E}^{-1} \mathbf{M E}=\Lambda
$$

where $\Lambda$ is a diagonal matrix constructed from the eigenvalues of $\mathbf{M}$.

At any point in a storage ring, a beam distribution $\Sigma$ that is properly matched to the lattice is invariant under a transformation given by the single-turn matrix $\mathbf{M}$ at that point

$$
\mathbf{x} \rightarrow \mathbf{M x}, \quad \Sigma \rightarrow \mathbf{M} \Sigma \mathbf{M}^{\mathrm{T}}=\Sigma .
$$

From the symplectic condition (10) for the single-turn matrix $\mathbf{M}$, and the invariance of the matched distribution under a transformation given by $\mathbf{M}$ (20), it follows that

$$
(\Sigma \mathbf{S})^{-1} \mathbf{M}(\Sigma \mathbf{S})=\mathbf{M}
$$

This relationship is satisfied by any matrix $\mathbf{M}$ that can be written as

$$
\mathbf{M}=\tilde{\mathbf{E}} \tilde{\Lambda} \tilde{\mathbf{E}}^{-1},
$$

where $\tilde{\Lambda}$ is a diagonal matrix and $\tilde{\mathbf{E}}$ is a matrix constructed from the eigenvectors of $\Sigma \mathbf{S}$

$$
(\Sigma \mathbf{S}) \tilde{\mathbf{E}}=\tilde{\mathbf{E}} \mathbf{D}
$$

with $\mathbf{D}$ the diagonal matrix constructed from the eigenvalues of $\Sigma \mathbf{S}$. But the diagonalization of $\mathbf{M}$ is uniquely given by the eigensystem of $\mathbf{M}$. Hence, we must have

$$
\begin{aligned}
\tilde{\mathbf{E}} & =\mathbf{E}, \\
\tilde{\Lambda} & =\Lambda .
\end{aligned}
$$

In other words, the eigenvectors of $\mathbf{M}$ are the same as the eigenvectors of $\Sigma \mathbf{S}$.

\section{B. Eigenvalues of $\Sigma S$}

Another useful result is that the eigenvalues of $\Sigma \mathbf{S}$ are just the beam emittances. To prove this, we first note that the eigenvalues of $\mathbf{\Sigma S}$ are invariant under a symplectic transformation of the phase-space coordinates

$$
\mathbf{x} \rightarrow \mathbf{U x}, \quad \Sigma \mathbf{S} \rightarrow \mathbf{U} \Sigma \mathbf{U}^{\mathrm{T}} \mathbf{S}=\mathbf{U} \Sigma \mathbf{S} \mathbf{U}^{-1} .
$$

Here, $\mathbf{U}$ is any symplectic matrix, and the final equality follows from the fact that $\mathbf{U}$ is symplectic. Note that $\Sigma \mathbf{S}$ and $\mathbf{U} \Sigma \mathbf{S U}^{-1}$ have the same eigenvalues (this is true for any matrices $\mathbf{U}$ and $\Sigma \mathbf{S}$ ). Now let $\mathbf{U}$ be the symplectic matrix that puts the single-turn matrix $\mathbf{M}$ into normal form

$$
\mathbf{U M U}^{-1}=\mathbf{R}
$$

where $\mathbf{R}$ is a rotation matrix. We shall give an explicit construction of $\mathbf{U}$ in Sec. III D. In principle, we can associate $\mathbf{U}$ with some beam line that decouples the beam. The beam line does not have to form a physical part of the storage ring; it only needs to exist in principle. Since the single-turn matrix at the end of the beam line associated with $\mathbf{U}$ is simply a rotation, the matched beam distribution matrix $\Sigma_{\mathbf{U}}=\mathbf{U} \Sigma \mathbf{U}^{\mathrm{T}}$ at this point is diagonal

$$
\Sigma_{\mathbf{U}}=\left(\begin{array}{cccccc}
\left\langle x^{2}\right\rangle & & & & & \\
& \left\langle p_{x}^{2}\right\rangle & & & & \\
& & \left\langle y^{2}\right\rangle & & & \\
& & & \left\langle p_{y}^{2}\right\rangle & & \\
& & & & \left\langle z^{2}\right\rangle & \\
& & & & & \left\langle\delta^{2}\right\rangle
\end{array}\right) .
$$

By solving the characteristic equation

$$
\operatorname{det}\left(\Sigma_{\mathbf{U}} \mathbf{S}-\lambda \mathbf{I}\right)=0
$$

we find that the eigenvalues of $\Sigma_{\mathbf{U}} \mathbf{S}$ are $\pm i \epsilon_{x}, \pm i \epsilon_{y}$ and $\pm i \epsilon_{z}$, where

$$
\begin{aligned}
& \epsilon_{x}=\sqrt{\left\langle x^{2}\right\rangle\left\langle p_{x}^{2}\right\rangle} \\
& \epsilon_{y}=\sqrt{\left\langle y^{2}\right\rangle\left\langle p_{y}^{2}\right\rangle}, \\
& \epsilon_{z}=\sqrt{\left\langle z^{2}\right\rangle\left\langle\delta^{2}\right\rangle}
\end{aligned}
$$

are the rms beam emittances. But since the eigenvalues of $\Sigma \mathbf{S}$ are preserved under any symplectic transformation of the phase-space coordinates, the eigenvalues of $\Sigma \mathbf{S}$ must also be $\pm i \epsilon_{k}$ where $k=\mathrm{I}$, II, or III (each value of $k$ corresponding to a different degree of freedom). The diagonalization of $\Sigma \mathbf{S}$ is

$$
\mathbf{E}^{-1}(\Sigma \mathbf{S}) \mathbf{E}=\mathbf{D}=\left(\begin{array}{lll}
i \overline{\mathbf{S}}_{2} \epsilon_{\mathrm{I}} & & \\
& i \overline{\mathbf{S}}_{2} \epsilon_{\mathrm{II}} & \\
& & i \overline{\mathbf{S}}_{2} \epsilon_{\mathrm{III}}
\end{array}\right) .
$$

To summarize, the single-turn matrix $\mathbf{M}$ and the matrix $\Sigma \mathbf{S}$ have the same eigenvectors $\mathbf{E}$, but different eigenvalues

$$
\begin{aligned}
\mathbf{M} & =\mathbf{E} \Lambda \mathbf{E}^{-1}, \\
\Sigma \mathbf{S} & =\mathbf{E} \mathbf{D} \mathbf{E}^{-1} .
\end{aligned}
$$

The eigenvalues $\Lambda$ of $\mathbf{M}$ are related to the tunes of the lattice, and the eigenvalues $\mathbf{D}$ of $\Sigma \mathbf{S}$ are related to the beam emittances.

\section{Actions and emittances}

We can construct quadratic functions of the phase-space coordinates of a single particle 


$$
J=\frac{1}{2} \mathbf{x}^{\mathrm{T}} \mathbf{A} \mathbf{x}
$$

where $\mathbf{A}$ is a symmetric matrix. If $\mathbf{A}$ satisfies

$$
\mathbf{M}^{\mathrm{T}} \mathbf{A} \mathbf{M}=\mathbf{A}
$$

then the quantity $J$ is invariant under the single-turn map

$$
\mathbf{x} \rightarrow \mathbf{M x}, \quad J \rightarrow J .
$$

With the additional constraint

$$
\operatorname{det} \mathbf{A}=1
$$

$J$ is an action of the particle. In 3 degrees of freedom, there are three possible symmetric matrices $\mathbf{A}$ that satisfy Eqs. (34) and (36), and hence three actions. Generally, two of the actions are associated with transverse motion (the betatron actions) and one with longitudinal motion (the synchrotron action).

Now consider again the transformation $\mathbf{U}$ that transforms the single-turn matrix $\mathbf{M}$ into a rotation $\mathbf{R}$. We define

$$
\mathbf{A}_{\mathbf{U}}=\left(\mathbf{U}^{-1}\right)^{\mathrm{T}} \mathbf{A} \mathbf{U}^{-1},
$$

which, from Eqs. (26) and (34) must satisfy

$$
\mathbf{R}^{\mathrm{T}} \mathbf{A}_{\mathbf{U}} \mathbf{R}=\mathbf{A}_{\mathbf{U}}
$$

In general, Eq. (38) has three solutions:

$$
\mathbf{A}_{\mathbf{U}}^{k}=\mathbf{T}^{k}
$$

where

$$
\begin{aligned}
\mathbf{T}^{\mathrm{I}} & =\left(\begin{array}{lll}
\mathbf{I}_{2} & & \\
& \mathbf{0}_{2} & \\
& & \mathbf{0}_{2}
\end{array}\right), \\
\mathbf{T}^{\mathrm{II}} & =\left(\begin{array}{lll}
\mathbf{0}_{2} & & \\
& \mathbf{I}_{2} & \\
& & \mathbf{0}_{2}
\end{array}\right), \\
\mathbf{T}^{\mathrm{III}} & =\left(\begin{array}{lll}
\mathbf{0}_{2} & & \\
& \mathbf{0}_{2} & \\
& & \mathbf{I}_{2}
\end{array}\right) .
\end{aligned}
$$

It follows that, after the transformation $\mathbf{U}$

$$
\begin{aligned}
\left\langle J_{\mathrm{I}}\right\rangle & =\frac{1}{2}\left\langle x^{2}\right\rangle+\frac{1}{2}\left\langle p_{x}^{2}\right\rangle, \\
\left\langle J_{\mathrm{II}}\right\rangle & =\frac{1}{2}\left\langle y^{2}\right\rangle+\frac{1}{2}\left\langle p_{y}^{2}\right\rangle, \\
\left\langle J_{\mathrm{III}}\right\rangle & =\frac{1}{2}\left\langle z^{2}\right\rangle+\frac{1}{2}\left\langle\delta^{2}\right\rangle .
\end{aligned}
$$

We also note that under the transformation $\mathbf{U}$, the matched distribution becomes

$$
\Sigma_{\mathbf{U}}=\left(\begin{array}{ccc}
\mathbf{I}_{2} \epsilon_{\mathrm{I}} & & \\
& \mathbf{I}_{2} \epsilon_{\mathrm{II}} & \\
& & \mathbf{I}_{2} \epsilon_{\mathrm{III}}
\end{array}\right) .
$$

So from Eqs. (41) and (42) we have

$$
\epsilon_{k}=\left\langle J_{k}\right\rangle
$$

Since the actions $J_{k}$ of the individual particles and the emittances $\boldsymbol{\epsilon}_{k}$ of the beam are invariant under any transformation $\mathbf{U}$, Eq. (43) must be true at any point in the beam line.

\section{Lattice functions}

Recall the matrix $\mathbf{E}$ that is constructed from the eigenvectors of either the single-turn matrix $\mathbf{M}$, or $\Sigma \mathbf{S}$ where $\Sigma$ is the matrix of second-order moments of the beam distribution. It is convenient to define a set of lattice functions that describe the matrix $\mathbf{E}$ in a physically meaningful way: these lattice functions will describe the linear optics of the lattice.

To derive the desired lattice functions, we first construct a real matrix $\mathbf{N}$ from $\mathbf{E}$

$$
\mathbf{N}=\mathbf{E Q},
$$

where

$$
\begin{aligned}
\mathbf{Q} & =\left(\begin{array}{lll}
\mathbf{Q}_{2} & & \\
& \mathbf{Q}_{2} & \\
& & \mathbf{Q}_{2}
\end{array}\right), \\
\mathbf{Q}_{2} & =\frac{1}{\sqrt{2}}\left(\begin{array}{cc}
1 & i \\
1 & -i
\end{array}\right) .
\end{aligned}
$$

With the normalization (14) of the eigenvectors, $\mathbf{N}$ is symplectic. Using $\mathbf{N}$, we can transform the single-turn matrix $\mathbf{M}$ into block-diagonal form

$$
\begin{aligned}
\mathbf{N}^{-1} \mathbf{M N} & =\mathbf{R}\left(\mu_{\mathrm{I}}, \mu_{\mathrm{II}}, \mu_{\mathrm{III}}\right) \\
& =\left(\begin{array}{lll}
\mathbf{R}_{2}\left(\mu_{\mathrm{I}}\right) & \\
& \mathbf{R}_{2}\left(\mu_{\mathrm{II}}\right) & \\
& & \mathbf{R}_{2}\left(\mu_{\mathrm{III}}\right)
\end{array}\right),
\end{aligned}
$$

where $\mu_{k}=2 \pi \nu_{k}$, and $\mathbf{R}_{2}(\theta)$ is the $2 \times 2$ rotation matrix

$$
\mathbf{R}_{2}(\theta)=\left(\begin{array}{cc}
\cos \theta & \sin \theta \\
-\sin \theta & \cos \theta
\end{array}\right) .
$$

Note that Eq. (44) gives us an explicit construction for the matrix $\mathbf{U}$ that we previously used to transform the singleturn map into a pure rotation

$$
\mathbf{U}=\mathbf{N}^{-1} .
$$

We now construct a vector $\mathbf{J}$ from the phase-space vector of a single particle

$$
\mathbf{J}=\mathbf{N}^{-1} \mathbf{x}
$$

Under a transformation given by the single-turn matrix, we have

$$
\mathbf{J}=\mathbf{N}^{-1} \mathbf{x} \rightarrow \mathbf{N}^{-1} \mathbf{M x}=\mathbf{N}^{-1} \mathbf{M N N}^{-1} \mathbf{x}=\mathbf{R J} .
$$

If $\mathbf{J}$ is written in the form 


$$
\mathbf{J}=\left(\begin{array}{c}
\sqrt{2 J_{\mathrm{I}}} \cos \phi_{\mathrm{I}} \\
-\sqrt{2 J_{\mathrm{I}}} \sin \phi_{\mathrm{I}} \\
\sqrt{2 J_{\mathrm{II}}} \cos \phi_{\mathrm{II}} \\
-\sqrt{2 J_{\mathrm{II}}} \sin \phi_{\mathrm{II}} \\
\sqrt{2 J_{\mathrm{III}}} \cos \phi_{\mathrm{III}} \\
-\sqrt{2 J_{\mathrm{III}}} \sin \phi_{\mathrm{III}}
\end{array}\right),
$$

then Eq. (50) shows that the actions $J_{k}$ are invariant under the single-turn matrix, and the angles $\phi_{k}$ transform as

$$
\mathbf{x} \rightarrow \mathbf{M x}, \quad \phi_{k} \rightarrow \phi_{k}+\mu_{k} .
$$

To make the connection with the invariant action introduced in Eq. (33), we note that

$$
\frac{1}{2} \mathbf{x}^{\mathrm{T}} \mathbf{A}^{k} \mathbf{x}=\frac{1}{2} \mathbf{J}^{\mathrm{T}} \mathbf{N}^{\mathrm{T}} \mathbf{A}^{k} \mathbf{N} \mathbf{J}=\frac{1}{2} \mathbf{J}^{\mathrm{T}} \mathbf{T}^{k} \mathbf{J}=J_{k},
$$

where we have used Eqs. (37), (39), and (48). From the definition of the emittance (43) and assuming that the angles of different particles in the beam are uncorrelated, we can write

$$
\left\langle\mathbf{J J}^{\mathrm{T}}\right\rangle=\sum_{k=\mathrm{I}, \mathrm{II}, \mathrm{III}} \mathbf{T}^{k} \boldsymbol{\epsilon}_{k} .
$$

It then follows that the beam distribution can be written

$$
\Sigma=\left\langle\mathbf{x} \mathbf{x}^{\mathrm{T}}\right\rangle=\mathbf{N}\left\langle\mathbf{J} \mathbf{J}^{\mathrm{T}}\right\rangle \mathbf{N}^{\mathrm{T}}=\sum_{k} \mathbf{N T}^{k} \mathbf{N}^{\mathrm{T}} \boldsymbol{\epsilon}_{k} .
$$

If we define three matrices

$$
\mathbf{B}^{k}=\mathbf{N} \mathbf{T}^{k} \mathbf{N}^{\mathrm{T}}=\mathbf{E} \overline{\mathbf{T}}^{k} \mathbf{E}^{\mathrm{T}},
$$

where

$$
\begin{aligned}
\overline{\mathbf{T}}^{\mathrm{I}} & =\left(\begin{array}{lll}
\overline{\mathbf{I}}_{2} & & \\
& \mathbf{0}_{2} & \\
& & \mathbf{0}_{2}
\end{array}\right), \\
\overline{\mathbf{T}}^{\mathrm{II}} & =\left(\begin{array}{lll}
\mathbf{0}_{2} & & \\
& \overline{\mathbf{I}}_{2} & \\
& & \mathbf{0}_{2}
\end{array}\right), \\
\overline{\mathbf{T}}^{\mathrm{III}} & =\left(\begin{array}{lll}
\mathbf{0}_{2} & & \\
& \mathbf{0}_{2} & \\
& & \overline{\mathbf{I}}_{2}
\end{array}\right)
\end{aligned}
$$

then we can write

$$
\Sigma=\sum_{k} \mathbf{B}^{k} \boldsymbol{\epsilon}_{k}
$$

Note that Eq. (56) may also be written

$$
\begin{aligned}
& \beta_{i j}^{\mathrm{I}}=n_{i 1} n_{j 1}+n_{i 2} n_{j 2}, \\
& \beta_{i j}^{\mathrm{II}}=n_{i 3} n_{j 3}+n_{i 4} n_{j 4}, \\
& \beta_{i j}^{\mathrm{III}}=n_{i 5} n_{j 5}+n_{i 6} n_{j 6},
\end{aligned}
$$

where the quantities $n_{i j}$ are the components of the matrix $\mathbf{N}$. We identify the elements $\beta_{i j}^{k}$ of $\mathbf{B}^{k}$ as the lattice functions that relate the beam emittances to the beam distribution. In Sec. IIIE we show that in the uncoupled case, the nonzero elements of $\mathbf{B}^{k}$ correspond to the usual
Twiss parameters. We note that an equation corresponding to Eq. (58) was obtained by Chao [8] in an analysis of the equilibrium beam distribution in an electron storage ring.

The lattice functions defined in Eq. (56) can be used to parameterize the single-turn matrix. First, we use Eq. (31) to write

$$
\mathbf{E}^{-1} \mathbf{M E}=\Lambda=\sum_{k}\left(\mathbf{T}^{k} \cos \mu_{k}+i \overline{\mathbf{T}}^{k} \mathbf{S} \sin \mu_{k}\right) .
$$

Using Eqs. (18) and (56) we find

$$
\left(\mathbf{B}^{k} \mathbf{S}\right)^{2}=-\mathbf{E}\left(\overline{\mathbf{T}}^{k} \mathbf{S}\right)^{2} \mathbf{E}^{-1}=-\mathbf{E} \mathbf{T}^{k} \mathbf{E}^{-1} .
$$

It then follows that we can write the single-turn matrix $\mathbf{M}$ in the form

$$
\mathbf{M}=\sum_{k=\mathrm{I}, \mathrm{II}, \mathrm{III}}\left(\mathbf{B}^{k} \mathbf{S} \sin \mu_{k}-\left(\mathbf{B}^{k} \mathbf{S}\right)^{2} \cos \mu_{k}\right) .
$$

\section{E. Special case: uncoupled lattices}

In the special case that the single-turn matrix is block diagonal, the motion is uncoupled in the sense that the coordinate in any plane is determined by just one of the three actions. If we identify $k=\mathrm{I}$ with the horizontal plane, and $k=$ II with the vertical plane, then there are simple relationships between the lattice functions defined in Eq. (56) and the familiar Twiss parameters. In the horizontal plane

$$
\begin{aligned}
& \beta_{x}=\beta_{11}^{\mathrm{I}} \\
& \alpha_{x}=-\beta_{12}^{\mathrm{I}}, \\
& \gamma_{x}=\beta_{22}^{\mathrm{I}},
\end{aligned}
$$

and in the vertical plane

$$
\begin{aligned}
\beta_{y} & =\beta_{33}^{\text {II }}, \\
\alpha_{y} & =-\beta_{34}^{\text {II }} \\
\gamma_{y} & =\beta_{44}^{\text {II }} .
\end{aligned}
$$

In a storage ring, the single-turn matrix is usually not completely block diagonal, but the transverse planes are coupled to the longitudinal plane through the dispersion. For example, the horizontal coordinate of a particle is given by

$$
x=\sqrt{2 \beta_{x} J_{x}} \cos \phi_{x}+\eta_{x} \delta,
$$

where $\eta_{x}$ is the horizontal dispersion. It follows that

$$
\langle x \delta\rangle=\eta_{x}\left\langle\delta^{2}\right\rangle .
$$

In terms of the lattice functions defined in Eq. (56) we can write

$$
\begin{aligned}
& \langle x \delta\rangle=\beta_{16}^{\mathrm{III}} \epsilon_{\mathrm{III}}, \\
& \left\langle\delta^{2}\right\rangle=\beta_{66}^{\mathrm{III}} \epsilon_{\mathrm{III}} .
\end{aligned}
$$

It immediately follows that the horizontal dispersion is 
given by

$$
\eta_{x}=\frac{\beta_{16}^{\mathrm{III}}}{\beta_{66}^{\mathrm{III}}}
$$

Similarly, the dispersion in the vertical plane is given by

$$
\eta_{y}=\frac{\beta_{36}^{\mathrm{III}}}{\beta_{66}^{\mathrm{III}}}
$$

\section{TRANSPORT LINES}

In a storage ring, the matched distribution is defined by the condition that the beam distribution matrix be invariant under the transformation given by the single-turn matrix. In a transport line (i.e., any nonperiodic beam line), there is no corresponding requirement, and we must start instead from some assumed distribution. Given the second-order moments $\Sigma$ of the distribution at some point in the beam line, we can find the corresponding lattice functions by constructing the eigenvectors $\mathbf{e}_{ \pm k}$ of $\Sigma \mathbf{S}$, and proceeding as in Sec. III D. Alternatively, we can assume values for the lattice functions at some point, and construct the distribution using Eq. (58). The question then is how we propagate the lattice functions (or, equivalently, the beam distribution matrix) along the beam line.

\section{A. Propagating the lattice functions}

Propagating the beam distribution is simple. We write the transfer matrix from position $s_{1}$ to position $s_{2}$ in a beam line as $\mathbf{M}_{21}$ :

$$
\mathbf{x}_{2}=\mathbf{M}_{21} \mathbf{x}_{1} \text {. }
$$

From Eq. (1), it follows that $\Sigma$ transforms as

$$
\Sigma_{2}=\mathbf{M}_{21} \Sigma_{1} \mathbf{M}_{21}^{\mathrm{T}} \text {. }
$$

Clearly, it is possible to derive the lattice functions at $s_{2}$ by finding the eigenvectors of $\Sigma_{2}$. However, since the lattice functions are independent of the beam emittances, we can construct an artificial distribution at $s_{1}$ from the known lattice functions at that point

$$
\Sigma^{k}\left(s_{1}\right)=\mathbf{B}^{k}\left(s_{1}\right) .
$$

The matrix $\Sigma^{k}$ describes the distribution of a beam that has $\epsilon_{k}=1$ for some chosen value of $k$, and zero emittances in the other 2 degrees of freedom. It then follows that the lattice functions are propagated along the beam line simply by calculating the matrix product:

$$
\mathbf{B}^{k}\left(s_{2}\right)=\mathbf{M}_{21} \mathbf{B}^{k}\left(s_{1}\right) \mathbf{M}_{21}^{\mathrm{T}} \text {. }
$$

\section{B. Phase advance}

It is often useful to know the phase advance between different points of the beam line. We first observe that there is a degeneracy in the matrix $\mathbf{N}$ that puts the matrix $\Sigma \mathbf{S}$ into block-diagonal form. The degeneracy in $\mathbf{N}$ corresponds to the fact that the absolute phase at any point is not determined: given two points in the beam line, only the phase advance between them is significant, since the phase advance gives the change in the angle, a dynamical variable, of any particle traveling from one point to the next. To fix a reference point, we must impose some conditions on $\mathbf{N}$ to remove the degeneracy. There are many ways to do this; one possible set of conditions, corresponding to the conventions used for uncoupled motion, may be written

$$
n_{12}=n_{34}=n_{56}=0 \text {. }
$$

In general, it is possible to find a rotation matrix $\mathbf{R}$ that can be used to transform $\mathbf{N}$ such that these conditions are satisfied; a matrix transformed in this way retains the defining property of block-diagonalizing $\Sigma \mathbf{S}$. We denote the "standardized" version of $\mathbf{N}$ by $\overline{\mathbf{N}}$.

From the standardized matrix $\overline{\mathbf{N}}_{1}$ that puts $\Sigma_{1} \mathbf{S}$ into block-diagonal form, we can construct

$$
\mathbf{N}_{2}=\mathbf{M}_{21} \overline{\mathbf{N}}_{1} \text {. }
$$

It is straightforward to show that $\mathbf{N}_{2}$ puts $\Sigma_{2} \mathbf{S}$ into blockdiagonal form; however, in general, it will not be standardized, i.e., the conditions (75) will not be satisfied. To perform the standardization, we simply apply a rotation

$$
\overline{\mathbf{N}}_{2}=\mathbf{N}_{2} \mathbf{R}^{-1}=\mathbf{M}_{21} \overline{\mathbf{N}}_{1} \mathbf{R}^{-1},
$$

where

$$
\mathbf{R}=\mathbf{R}\left(\Delta \phi_{\mathrm{I}}, \Delta \phi_{\mathrm{II}}, \Delta \phi_{\text {III }}\right)=\overline{\mathbf{N}}_{2}^{-1} \mathbf{M}_{21} \overline{\mathbf{N}}_{1} .
$$

The rotation angles $\Delta \phi_{k}$ are the required phase advances.

\section{APPLICATIONS}

The principal value of the lattice functions defined by Eq. (56) is that they provide a conceptually simple way to describe the linear optics in a coupled system. We also find that useful formulas can be expressed in an elegant and concise way using these functions. In this section, we give examples of both these benefits.

\section{A. Phase advance and lattice functions}

In an uncoupled lattice, the horizontal phase advance is related to the horizontal beta function by

$$
\frac{d \phi_{x}}{d s}=\frac{1}{\beta_{x}}
$$

(and similarly for the vertical plane). We can find an analogous expression in the case of coupled optics. For simplicity, we consider the phase advance in the plane $k=$ I; the analysis is easily generalized to the other planes.

First, we find from Eqs. (75)-(77) that the phase advance resulting from the transformation $\mathbf{M}_{21}$ is given by 


$$
\tan \Delta \phi_{\mathrm{I}}=\frac{n_{12}^{(2)}}{n_{11}^{(2)}}
$$

where $n_{i j}^{(2)}$ are the elements of $\mathbf{N}_{2} . \Delta \phi_{\mathrm{I}}$ is just the rotation angle needed to satisfy the standard phase choice (75). In an accelerator beam line, the form of $\mathbf{M}_{21}$ is constrained by Maxwell's and Hamilton's equations. By considering the linear transfer matrices for drift spaces, dipoles, normal and skew quadrupoles and solenoids, we find that particle transport over a short distance $\Delta s$ in any of these linear elements has the result (to first-order in $\Delta s$ ):

$$
\frac{n_{12}^{(2)}}{n_{11}^{(2)}}=\frac{\bar{n}_{22}+\frac{1}{2} k_{s} \bar{n}_{32}}{\bar{n}_{11}} \Delta s
$$

where $k_{s}$ is the solenoid field strength normalized to the beam rigidity, and $\bar{n}_{i j}$ are the elements of $\overline{\mathbf{N}}_{1}$. Combining Eqs. (80) and (81), and taking the limit $\Delta s \rightarrow 0$, we find

$$
\frac{d \phi_{\mathrm{I}}}{d s}=\frac{\bar{n}_{22}+\frac{1}{2} k_{s} \bar{n}_{32}}{\bar{n}_{11}}
$$

Using Eq. (56), we can write Eq. (82) in terms of the lattice functions. The result is

$$
\frac{d \phi_{\mathrm{I}}}{d s}=\frac{1}{\beta_{11}^{\mathrm{I}}}\left(\frac{\Delta_{1122}^{\mathrm{I}}+\frac{1}{2} k_{s} \Delta_{1123}^{\mathrm{I}}}{\sqrt{\Delta_{1122}^{\mathrm{I}}}}\right),
$$

where we have defined

$$
\Delta_{i j i^{\prime} j^{\prime}}^{k}=\beta_{i j}^{k} \beta_{i^{\prime} j^{\prime}}^{k}-\beta_{i j^{\prime}}^{k} \beta_{i^{\prime} j}^{k} .
$$

Note that the solenoid strength enters explicitly in Eq. (83); otherwise, the right-hand side is expressed purely in terms of the lattice functions. In the uncoupled case, $k_{s}=0$ and $\Delta_{1122}^{\mathrm{I}}=1$, and we recover Eq. (79).

\section{B. Tune shift from perturbative focusing error}

It is often useful to know the tune shift that results in a storage ring from a perturbative focusing error at some point in the lattice. Let us consider a focusing error that may be represented by the transfer matrix

$$
\mathbf{K}=\left(\begin{array}{cccccc}
1 & 0 & 0 & 0 & 0 & 0 \\
\kappa_{11} & 1 & \kappa_{13} & 0 & \kappa_{15} & 0 \\
0 & 0 & 1 & 0 & 0 & 0 \\
\kappa_{31} & 0 & \kappa_{33} & 1 & \kappa_{35} & 0 \\
0 & 0 & 0 & 0 & 1 & 0 \\
\kappa_{51} & 0 & \kappa_{53} & 0 & \kappa_{55} & 1
\end{array}\right)
$$

Note that the symplectic condition on $\mathbf{K}$ requires that

$$
\begin{aligned}
& \kappa_{31}=\kappa_{13}, \\
& \kappa_{51}=\kappa_{15}, \\
& \kappa_{53}=\kappa_{35} .
\end{aligned}
$$

Let $\mathbf{M}$ be the single-turn transfer matrix in the absence of the focusing error, at the location of the error. In the presence of the focusing error, the single-turn matrix may be expressed as

$$
\mathbf{M} \mathbf{K}=\mathbf{N R} \mathbf{N}^{-1} \mathbf{K}=\mathbf{N R} \mathbf{N}^{-1} \mathbf{K N N}^{-1},
$$

where $\mathbf{N}$ is the normalizing transformation for $\mathbf{M}$, and $\mathbf{R}$ is a block-diagonal rotation matrix. The rotation angles in $\mathbf{R}$ are the lattice tunes multiplied by $2 \pi$. $\mathbf{N}$ determines the lattice functions; to find the perturbative tune shift to first order in the focusing error, we can neglect the variation in the lattice functions from the focusing error, and assume that $\mathbf{N}$ normalizes $\mathbf{M K}$ as well as $\mathbf{M}$. Hence, we write

$$
\mathbf{M K}=\mathbf{N R} \cdot \Delta \mathbf{R} \cdot \mathbf{N}^{-1},
$$

where

$$
\Delta \mathbf{R}=\mathbf{N}^{-1} \mathbf{K N}
$$

is (close to) a rotation matrix, with rotation angles equal to the tune shifts resulting from the focusing error.

Since $\mathbf{N}$ is symplectic, we have

$$
\mathbf{S} \cdot \Delta \mathbf{R}=\mathbf{N}^{\mathrm{T}} \mathbf{S K N} \text {. }
$$

For small tune shifts, the tune shifts may be obtained from the diagonal elements of $\mathbf{S} \cdot \Delta \mathbf{R}$

$$
\begin{aligned}
\Delta \mu_{\mathrm{I}} & \approx \frac{1}{2}\left(\left[\mathbf{N}^{\mathrm{T}} \mathbf{S K N}\right]_{11}+\left[\mathbf{N}^{\mathrm{T}} \mathbf{S} \mathbf{K} \mathbf{N}\right]_{22}\right), \\
\Delta \mu_{\mathrm{II}} & \approx \frac{1}{2}\left(\left[\mathbf{N}^{\mathrm{T}} \mathbf{S K N}\right]_{33}+\left[\mathbf{N}^{\mathrm{T}} \mathbf{S K N}\right]_{44}\right), \\
\Delta \mu_{\mathrm{III}} & \approx \frac{1}{2}\left(\left[\mathbf{N}^{\mathrm{T}} \mathbf{S K N}\right]_{55}+\left[\mathbf{N}^{\mathrm{T}} \mathbf{S K N}\right]_{66}\right) .
\end{aligned}
$$

Using Eq. (56), we find that for symplectic focusing errors of the form (85), the tune shifts can be expressed as

$$
\Delta \nu^{k} \approx-\frac{1}{4 \pi} \sum_{i, j=1,3,5} \beta_{i j}^{k} \kappa_{i j},
$$

where, as usual, $k=\mathrm{I}$,II,III. Equation (92) has been found (in a slightly different form) by Venturini [9] in the context of space-charge tune shifts in a coupled lattice.

In the special case of an uncoupled lattice, Eq. (92) reduces to the familiar form

$$
\begin{aligned}
& \Delta \nu_{x}=-\frac{1}{4 \pi} \beta_{x} \kappa_{x}, \\
& \Delta \nu_{y}=-\frac{1}{4 \pi} \beta_{y} \kappa_{y},
\end{aligned}
$$

for the horizontal and vertical planes, where $\kappa_{x}=\kappa_{11}$, and $\kappa_{y}=\kappa_{33}$.

\section{Flat-beam to round-beam transformer}

The space-charge tune shifts in large lattices at low energy can become large when the vertical emittance is small, as is the case in some designs for linear collider damping rings, for example [10]. Coupling bumps have been proposed as a way to make the vertical beam size large even when the vertical emittance is small, thus re- 
ducing the charge density and mitigating potentially harmful space-charge effects. The basic concepts have been described by Derbenev et al.; see, for example, [11]. Here, we briefly describe the required transformations, and give the coupled lattice functions in an implementation in one design for a $16 \mathrm{~km}$ lattice for the ILC damping rings [12].

We treat only the transverse degrees of freedom. Consider a point in the lattice where the (uncoupled) Twiss beta functions are equal in the horizontal and vertical planes, $\beta_{x}=\beta_{y}=\beta$, the Twiss alpha functions are zero and there is no coupling. The $4 \times 4$ beam distribution matrix is given by

$$
\Sigma=\left(\begin{array}{cccc}
\beta \epsilon_{x} & 0 & 0 & 0 \\
0 & \frac{\epsilon_{x}}{\beta} & 0 & 0 \\
0 & 0 & \beta \epsilon_{y} & 0 \\
0 & 0 & 0 & \frac{\epsilon_{y}}{\beta}
\end{array}\right)
$$

Now we take a symplectic transformation $\mathbf{V}$

$$
\mathbf{V}=\tilde{\mathbf{R}}\left(-\frac{\pi}{4}\right) \mathbf{R}_{\beta}(\beta, \mu) \tilde{\mathbf{R}}\left(\frac{\pi}{4}\right)
$$

where $\tilde{\mathbf{R}}$ represents a rotation in coordinate space

$$
\tilde{\mathbf{R}}(\theta)=\left(\begin{array}{cccc}
\cos \theta & 0 & \sin \theta & 0 \\
0 & \cos \theta & 0 & \sin \theta \\
-\sin \theta & 0 & \cos \theta & 0 \\
0 & -\sin \theta & 0 & \cos \theta
\end{array}\right)
$$

and $\mathbf{R}_{\beta}$ represents a linear transformation in phase space (with different phase advances in the horizontal and vertical planes)

$$
\mathbf{R}_{\beta}(\beta, \mu)=\left(\begin{array}{cccc}
\cos \mu & \beta \sin \mu & 0 & 0 \\
-\frac{1}{\beta} \sin \mu & \cos \mu & 0 & 0 \\
0 & 0 & -\sin \mu & \beta \cos \mu \\
0 & 0 & -\frac{1}{\beta} \cos \mu & -\sin \mu
\end{array}\right)
$$

After the transformation $\mathbf{V}$, for any value of the phase advance $\mu$, the distribution matrix becomes

$$
\Sigma_{\mathbf{V}}=\mathbf{V} \Sigma \mathbf{V}^{\mathrm{T}}=\left(\begin{array}{cccc}
\frac{1}{2} \beta \epsilon_{+} & 0 & 0 & \frac{1}{2} \epsilon_{-} \\
0 & \frac{1}{2 \beta} \epsilon_{+} & -\frac{1}{2} \epsilon_{-} & 0 \\
0 & -\frac{1}{2} \epsilon_{-} & \frac{1}{2} \beta \epsilon_{+} & 0 \\
\frac{1}{2} \epsilon_{-} & 0 & 0 & \frac{1}{2 \beta} \epsilon_{+}
\end{array}\right),
$$

where $\epsilon_{ \pm}=\epsilon_{x} \pm \epsilon_{y}$. After the transformation, the horizontal and vertical beam sizes are equal, and the beam has no tilt. The required transformation can be achieved using a set of three equally spaced skew quadrupoles, with parameters

$$
\begin{aligned}
k_{1} L & =\frac{2 \sqrt{2}}{\beta}, \\
\alpha & =-\frac{1}{2}\left(1+\frac{1}{\sqrt{2}}\right), \\
d & =\frac{\beta}{2 \sqrt{1+\sqrt{2}}},
\end{aligned}
$$

where $k_{1} L$ is the integrated strength (normalized by the beam rigidity) of the central skew quadrupole, $\alpha k_{1} L$ is the normalized integrated strength of each of the outer two skew quadrupoles, and $d$ is the distance between the skew quadrupoles. The same transformation can be used to decouple the beam, as long as the phase advances of the two transverse modes are equal between the transformations.

Figure 1 shows an example of a flat-beam to round-beam transformation at one end of a long straight section in a design for a $16 \mathrm{~km}$ damping ring lattice for ILC. The straight section is $6 \mathrm{~km}$ long, and has high beta functions, to allow large separation between quadrupoles. The flatbeam to round-beam transformation is implemented at the entrance to the straight section, where a matching section is used to set the beta functions to $140 \mathrm{~m}$, and the alpha functions to zero. The coupling transformations are implemented using thin multipoles, with the exact strengths and separations given by Eqs. (99). The resulting lattice functions can be seen in Fig. 1: note that the skew quadrupoles are located at 2148, 2193, and $2238 \mathrm{~m}$. A second transformation (not shown) is used to decouple the beam at the exit of the long straight section. The lattice functions in Fig. 1 are the matched functions in the complete damping ring lattice, calculated in MERLIN [13] using the techniques described in Sec. III.

The effect of the flat-beam to round-beam transformation on the beam distribution is easily understood from Fig. 1, given the relationships between the beam distribution and the coupled lattice functions

$$
\begin{aligned}
& \left\langle x^{2}\right\rangle=\beta_{11}^{\mathrm{I}} \epsilon_{\mathrm{I}}+\beta_{11}^{\mathrm{II}} \epsilon_{\mathrm{II}}, \\
& \left\langle y^{2}\right\rangle=\beta_{33}^{\mathrm{I}} \epsilon_{\mathrm{I}}+\beta_{33}^{\mathrm{II}} \epsilon_{\mathrm{II}}, \\
& \langle x y\rangle=\beta_{13}^{\mathrm{I}} \epsilon_{\mathrm{I}}+\beta_{13}^{\mathrm{II}} \epsilon_{\mathrm{II}} .
\end{aligned}
$$

The middle plot in Fig. 1 shows that after the flat-beam to round-beam transformation, even with vanishing $\epsilon_{\mathrm{II}}$ the vertical beam size is comparable to the horizontal beam size, since $\epsilon_{\mathrm{I}}$ makes a significant contribution to $\left\langle y^{2}\right\rangle$. The bottom plot gives the correlation $\langle x y\rangle$, which is zero before the transformation, and remains small (though nonzero) after the transformation.

\section{Longitudinal focusing}

RF cavities provide longitudinal focusing analogous to the transverse focusing provided by quadrupoles. The lattice function $\beta_{55}^{\mathrm{III}}$ provides a natural analog of the transverse lattice functions $\beta_{x}$ and $\beta_{y}$, and describes the effect 


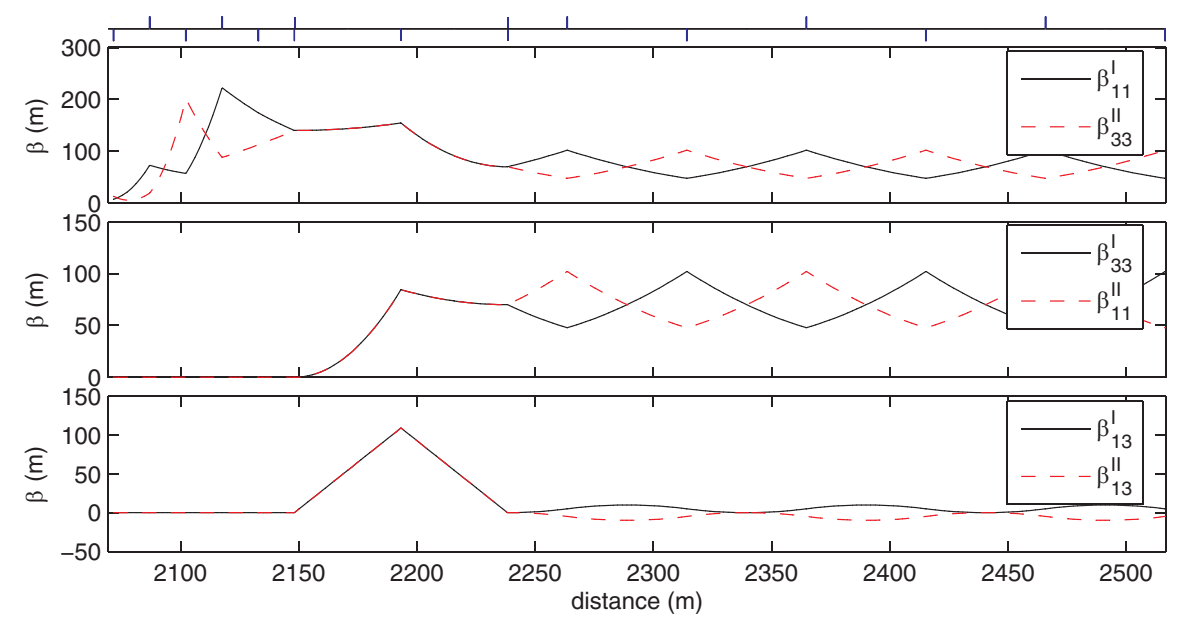

FIG. 1. (Color) Coupled lattice functions in a flat-beam to round-beam transformer.

of the longitudinal focusing on the bunch length, which varies around the ring. In most storage rings, the focusing is so weak that the variation in bunch length around the ring is small enough that it may be neglected, but in some regimes it may be significant. This occurs, for example, in the $16 \mathrm{~km}$ ILC damping ring previously referred to (Sec. VC), where the combination of large RF voltage and high momentum compaction leads to a large synchrotron tune.

Figure 2 shows the longitudinal lattice functions in part of the damping ring lattice. The section shown includes one arc (consisting of a bend through $270^{\circ}$ followed by a reverse bend through $-90^{\circ}$ ), followed by an RF section. The rest of the lattice consists of long straight sections, in which there are no longitudinal dynamics, and a second $\operatorname{arc/RF}$ section identical to the one shown. The lattice functions were calculated in MERLIN [13].

The interpretation of the longitudinal lattice functions is straightforward. In this case, the bunch length and energy spread have negligible dependence on the transverse emittances $\epsilon_{\mathrm{I}}$ and $\epsilon_{\mathrm{II}}$, so the longitudinal distributions can be written

$$
\begin{aligned}
\left\langle z^{2}\right\rangle & =\beta_{55}^{\mathrm{III}} \epsilon_{\mathrm{III}}, \\
\langle z \delta\rangle & =\beta_{56}^{\mathrm{III}} \epsilon_{\mathrm{III}}, \\
\left\langle\delta^{2}\right\rangle & =\beta_{66}^{\mathrm{III}} \epsilon_{\mathrm{III}} .
\end{aligned}
$$

The top plot in Fig. 2 shows that the rms bunch length varies by about $7 \%$ through the lattice. The middle plot shows the correlated energy spread.

\section{E. Calculating coupled lattice functions from phase-advance data}

A useful technique for characterizing the optics in a storage ring involves resonant excitation of low-amplitude betatron oscillations of a bunch, followed by the measurement of the trajectory of the bunch over a few hundred turns. The turn-by-turn readings at each BPM form a sine wave; the phase difference of the waves from any two BPMs gives the phase advance (in the plane with tune corresponding to the excitation frequency) between those BPMs. It is useful to be able to reconstruct the lattice

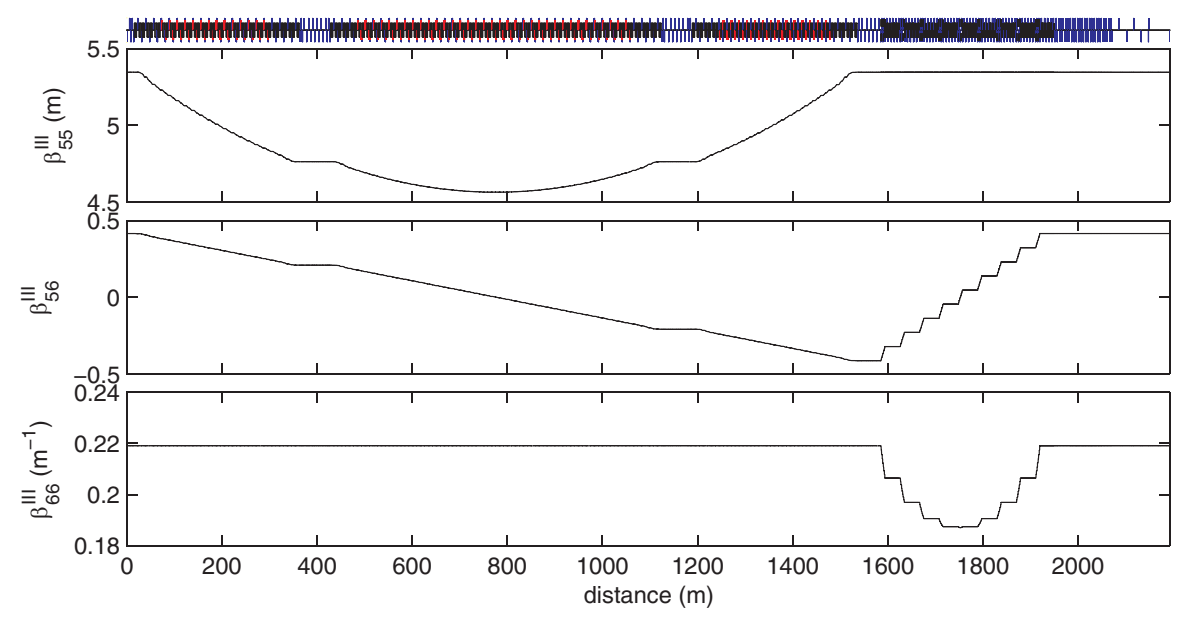

FIG. 2. (Color) Longitudinal lattice functions in part of a $16 \mathrm{~km}$ ILC damping ring lattice. 
functions from the phase-advance data. Techniques for this, which involve assumptions about the transfer matrices between BPMs, are well established for the uncoupled case [14-18]. Here, we consider how the phase-advance data may be used to construct the coupled lattice functions derived in Sec. III.

Let us first review the procedure used for an uncoupled beam line. Consider a point $s_{1}$ in the beam line: the phase advance $\Delta \phi_{21}$ from $s_{1}$ to some other point, $s_{2}$, along the beam line can be found from

$$
\cot \Delta \phi_{21}=\frac{m_{11}^{(21)}}{m_{12}^{(21)}} \beta-\alpha,
$$

where $m_{i j}^{(21)}$ are elements of $\mathbf{M}_{21}$ (the transfer matrix from $s_{1}$ to $s_{2}$ ) and $\beta$ and $\alpha$ are the lattice functions at $s_{1}$. Similarly, the phase advance $\Delta \phi_{31}$ from $s_{1}$ to a third point $s_{3}$ is found from

$$
\cot \Delta \phi_{31}=\frac{m_{11}^{(31)}}{m_{12}^{(31)}} \beta-\alpha .
$$

Conversely, if we know the phase advances $\Delta \phi_{21}$ and $\Delta \phi_{31}$ and the transfer matrices $\mathbf{M}_{21}$ and $\mathbf{M}_{31}$, then we can solve Eqs. (102) and (103) for the lattice functions $\beta$ and $\alpha$.

The technique generalizes easily to fully coupled motion, although because we need more lattice functions to describe the optics completely, we will need more data. For simplicity, let us consider only the transverse motion, and suppose that we measure the phase advance in the horizontal plane, i.e., by exciting a trajectory corresponding to the horizontal tune. In this case, the phase advance $\Delta \phi_{21}$ from $s_{1}$ to any point $s_{2}$ can be found from

$$
\cot \Delta \phi_{21}=\frac{n_{11}^{(2)}}{n_{12}^{(2)}}=\frac{\sum_{i=1}^{4} m_{1 i}^{(21)} \bar{n}_{i 1}}{\sum_{i=2}^{4} m_{1 i}^{(21)} \bar{n}_{i 2}},
$$

where $\bar{n}_{i j}$ are elements of the normalizing matrix $\overline{\mathbf{N}}_{1}$ at $s_{1}$, with $\bar{n}_{12}=0$, and $n_{i j}^{(2)}$ are elements of the normalizing matrix $\mathbf{N}_{2}$ at $s_{2}$; the phase advance $\Delta \phi_{21}$ is just the angle needed to transform $\mathbf{N}_{2}$ to satisfy $n_{12}=0$. Now we simply write Eq. (104) in the form

$$
\cot \Delta \phi_{21}\left(\sum_{i=2}^{4} m_{1 i}^{(21)} \bar{n}_{i 2}\right)-\sum_{i=1}^{4} m_{1 i}^{(21)} \bar{n}_{i 1}=0 .
$$

In general, given the phase advance and the transfer matrices from $s_{1}$ to six other points along the beam line, we can use Eq. (105) to construct a set of six simultaneous equations. The symplectic constraint on $\overline{\mathbf{N}}_{1}$ provides one additional equation, and we may then solve the complete system for the seven unknowns $\bar{n}_{i j}$, with $i=1, \ldots, 4$ and $j=1,2$ (and $\bar{n}_{12}=0$ ). The lattice functions are found from Eq. (59). In some cases, some or many of the transfer matrix elements may be zero, and it may not be possible to solve for all of the lattice functions. Usually, however, it will be possible to solve for the "in-plane" functions, $\beta_{11}^{\mathrm{I}}$ etc.

An example of the calculation of the lattice functions from phase-advance data in the PEP-II High-Energy Ring is shown in Fig. 3. The interaction point is at $733.1 \mathrm{~m}$, and the coupling extends out approximately $150 \mathrm{~m}$ on either side; only the lattice functions in the coupled section are

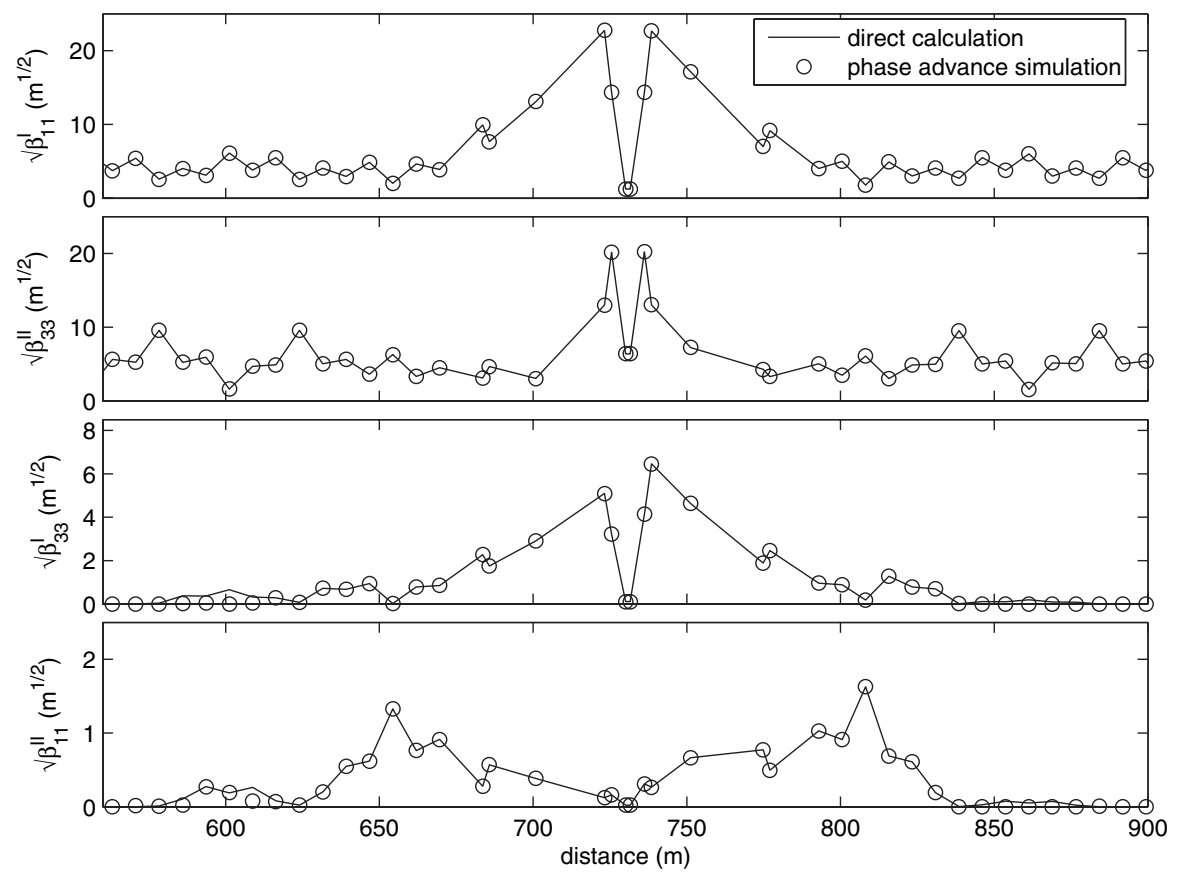

FIG. 3. Calculation of lattice functions from simulated phase-advance data in the PEP-II HER. 
shown. The top two plots show the square roots of the "inplane" lattice functions $\beta_{11}^{\mathrm{I}}$ and $\beta_{33}^{\mathrm{II}}$, while the bottom two plots show the square roots of the "cross-plane" lattice functions $\beta_{33}^{\mathrm{I}}$ and $\beta_{11}^{\mathrm{II}}$. The values calculated directly from the model using the methods of Sec. III are shown as solid lines; the values calculated from simulated phase-advance data in the model using Eqs. (59) and (105) are shown as circles. Note that the horizontal positions of the circles correspond to the locations of the BPMs. To calculate the lattice functions at each BPM from the phase-advance data, we use the phase advance from that BPM to the nearest three BPMs on either side. The values of some of the cross-plane lattice functions near the ends of the coupled section are not accurately determined, since the equations constructed from Eq. (105) are not wellconditioned in those cases. Where this situation occurs, it may be possible to improve accuracy by using the phaseadvance data to a different set of six BPMs than the nominal ones chosen.

\section{ACKNOWLEDGMENTS}

The author thanks Miguel Furman, David Sagan, Uli Wienands and Mark Woodley for useful discussions. This work was supported by the Director, Office of Science, High-Energy Physics, U.S. Department of Energy under Contract No. DE-AC02-05CH11231.

[1] D. Edwards and L. Teng, IEEE Trans. Nucl. Sci. 20, 3 (1973).

[2] D. Sagan and D. Rubin, Phys. Rev. ST Accel. Beams 2, 074001 (1999).
[3] Y. Luo, Phys. Rev. ST Accel. Beams 7, 124001 (2004).

[4] R. Calaga, R. Tomas, and A. Franchi, Phys. Rev. ST Accel. Beams 8, 034001 (2005).

[5] G. Ripken, DESY Report No. R1-70/04, 1970 (unpublished).

[6] H. Wiedemann, Particle Accelerator Physics II, Nonlinear and Higher-Order Beam Dynamics (Springer-Verlag, Berlin, 1995), p. 86.

[7] V. A. Lebedev and S. A. Bogacz, JLAB Report No. JLABACC-99-19 (unpublished).

[8] A. Chao, J. Appl. Phys. 50, 595 (1979).

[9] M. Venturini, LBNL Report No. LBNL-57664, 2005 (unpublished).

[10] W. Decking and R. Brinkmann, in Proceedings of the European Particle Accelerator Conference, Vienna, Austria, 2000 (EPS-IGA/CERN, Geneva, 2000), p. 1024.

[11] A. Burov, S. Nagaitsev, and Y. Derbenev, Fermilab Report No. FERMILAB-Pub-01/060-T, 2001 (unpublished).

[12] A. Wolski, LBNL Report No. LBNL-57045, 2005 (unpublished).

[13] N. J. Walker, computer code MERLIN; http://www.desy.de/ $\sim$ merlin/

[14] P. Castro-Garcia, Doctoral thesis, Universitat de Valencia, 1996 (unpublished).

[15] D. Sagan, R. Meller, R. Littauer, and D. Rubin, Phys. Rev. ST Accel. Beams 3, 092801 (2000).

[16] D. Sagan, Phys. Rev. ST Accel. Beams 3, 102801 (2000).

[17] U. Wienands, M.H. Donald, and M.E. Biagini, in Proceedings of the European Particle Accelerator Conference, Paris, France, 2002 (EPS-IGA/CERN, Geneva, 2002), p. 1202.

[18] M.H. Donald, T. M. Himel and M.S. Zelazny, in Proceedings of the European Particle Accelerator Conference, Lucerne, Switzerland, 2004 (EPS-IGA/ CERN, Geneva, 2004), p. 2840. 